\title{
Metodologías activas para el aprendizaje del instrumento de medición- micrómetro
}

\section{Active methodologies for learning the measuring instrument-micrometer}

\author{
Darwin Gregorio Chele Sancán \\ Edgar Gustavo Vera Puebla \\ Universidad Internacional del Ecuador, Ecuador
}

Autor para correspondencia: dachelesa@uide.edu.ec; edverapu@uide.edu.ec

Fecha de recepción: 15 de agosto de 2018 - Fecha de aceptación: 15 octubre de 2018

Resumen: El presente estudio tiene como objetivo promover el uso de metodologías activas para el aprendizaje del instrumento de medición directa, micrómetro, a la vez que se aplica su principio de funcionamiento, tornillo-tuerca. Lo descrito en este trabajo puede ser empleado para el sistema métrico y el sistema inglés de unidades $(0,01 \mathrm{~mm}$ y 0,001 pulgadas respectivamente), por medio de la ampliación de las escalas de graduación (las cuales estarán limitadas de acuerdo con el paso del elemento roscado que se utilice) tanto de la escala principal, grabadas en el cilindro exterior como la de la escala vernier, grabada en el tambor, cuya ampliación de las graduaciones dependerá del diámetro en la zona donde se las realice. También se realizó una breve introducción sobre Metrología, sistemas de medición y unidades de medida, así como del instrumento de medición utilizado en el presente estudio, además se expone una propuesta de cómo obtener la apreciación o resolución del instrumento de medición. Para alcanzar la validez de este estudio, y obtener un aprendizaje significativo y duradero, se empleó las siguientes metodologías activas de aprendizaje: el trabajo cooperativo, donde los estudiantes trabajan en conjunto para lograr un objetivo común y son responsables todos los que conforman el grupo, de que puedan aprender unos de otro, a la vez que desarrollan aptitudes propias de trabajo en grupo; el aprendizaje basado en problemas, donde se busca que el estudiante construya su conocimiento sobre problemas y casos de la vida real y el aprendizaje basado en proyectos, donde el estudiante desde sus inicios se enfrente a un aprendizaje activo por medio de proyectos de ingeniería simples, metodologías que permiten que el estudiante participe de manera activa y sea motivado en su proceso de formación como futuro profesional en ingeniería mediante una planificación adecuada de las actividades lúdicas en clases, guiados por el docente, con materiales significativos y conceptualmente de fácil comprensión. Para concretar el estudio, se procede a elaborar un proyecto de aplicación, el cual consiste en la elaboración de un elemento que represente la escala principal y la escala Vernier de un micrómetro en madera con sus respectivas graduaciones.

Palabras Claves: aprendizaje; metodologías; metrología; micrómetro; sistema de unidades

Abstract: The objective of this study is to promote the use of active methodologies for learning the direct measurement instrument, micrometer, while applying its operating principle, screw-nut. What is described in this article, it can be used for the Metric and the English systems of units ( $0.01 \mathrm{~mm}$ and 0.001 inches respectively), by means of the extension of the graduation scales 
(which will be limited according to the step of the threaded element used) of the main scale, recorded on the outer cylinder as well as on the vernier scale, engraved on the thimble, whose enlargement of the graduations will depend on the diameter in the area where they are made. A brief introduction was also made on Metrology, measurement systems and units of measure, as well as the measurement instrument used in the present study, as well as a proposal on how to obtain the assessment or resolution of the measurement instrument. In order to achieve the validity of this study, and obtain a meaningful and lasting learning, the following active learning methodologies were used: cooperative work, where students work together to achieve a common goal and they are responsible for all those who work in the group, they can learn from each other, while developing skills of group work; problem-based learning, where students are expected to build their knowledge with problems and real-life cases and project-based learning, where the student from the beginning are faced with active learning through simple engineering projects, methodologies which allow the students to participate actively and be motivated in their training process as a future professional in engineering through proper planning of group activities in classes, guided by the teacher, with meaningful materials conceptually easy to understand. To finalize the study, we proceed to develop an application project, which consists of the development of an element that represents the main scale and vernier scale of a micrometer in wood with their respective graduations.

Key Words: learning; methodologies; metrology; micrometer; unit system 


\section{Introducción}

\section{Problema}

El instrumento de medición micrómetro es muy utilizado en los talleres mecánicos, la manufactura y el negocio de autopartes, siendo de vital importancia debido a que los componentes de las máquinas o mecanismos que las conforman presentan tolerancias de fabricación que deben de ser satisfechas, por lo tanto, hay la necesidad de realizar mediciones con instrumentos que tengan un mínimo de error posible.

La enseñanza sobre la utilización, lectura y cuidados de los instrumentos de medición es un poco compleja, aún más a la hora de interpretar la medida que estas indican, debido a que la escala de graduación que presentan los instrumentos es pequeñas. Las TIC's permiten tener acceso a instrumentos de medición virtuales donde las escalas son ampliadas y ayudan realizar prácticas de lectura, estos sin embargo pueden ser distractores cuando no se hace un buen uso de los medios tecnológicos.

El estudiante logra el aprendizaje significativo cuando conecta los conocimientos previos con los nuevos conocimientos, guiados por el docente mediante clases magistrales y la interacción que se pueda generar entre docente-estudiante y entre estudiantes. El presente trabajo pretender generar el aprendizaje significativo a través de un aprendizaje basado en proyectos (ABPr), donde el estudiante de ingeniería desde sus inicios en la carrera enfrente un aprendizaje activo, guiado por el docente, a quien en la actualidad se le da un nuevo rol, el de facilitador, experimentando situaciones propias de un ingeniero, dejando el exceso de teoría para dar paso a la práctica (aprender haciendo).

\section{Metrología}

\section{Fundamentación teórica}

Literalmente metrología significa la ciencia de las medidas, en otras palabras la ciencia que se ocupa de las medidas, misma que se ha desarrollado en función de las necesidades de la sociedad en cada época. En la ingeniería se la utiliza para medir longitudes y ángulos y otras cantidades que están expresadas en estos términos. La metrología también establece las unidades de medida, es indispensable y juega un papel muy importante en nuestras vidas de manera directa o indirecta, una de las principales funciones de la metrología es establecer los estándares internacionales de medidas utilizados en la ciencia y la industria por todos los países en el mundo (Del Campo \& Robles, 2012; Raghavendra \& Krishnamurthy, 2013).

\section{Sistema de medidas y unidades}

El Sistema Internacional (SI) de Unidades y el Sistema Inglés o anglosajón de Unidades son utilizados actualmente en el área automotriz, el primero tiene como unidad fundamental el metro, el cual multiplicando o dividiendo se obtiene los múltiplos y submúltiplos respectivamente, siendo el milímetro $(\mathrm{mm})$ el más utilizado en este sistema de unidades. 


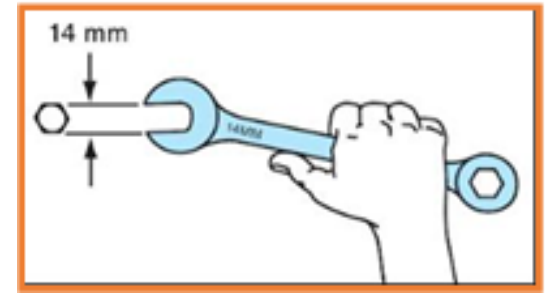

Figura 1. Medida de una llave mixta en milímetros.

Fuente: Basic Automotive Service and Systems.

La pulgada en el Sistema Inglés de Unidades es la más utilizada en el área automotriz, con los submúltiplos 0,001 y 0,0001 pulgadas, también se utiliza las fracciones de pulgadas, por ejemplo 1/8 - 1/4 - 7/16 - 1/2 ..... (García \& Fernández-Bravo, 2010; Chávez, Mejía, \& Pacheco, 2009; Escamilla, 2014).

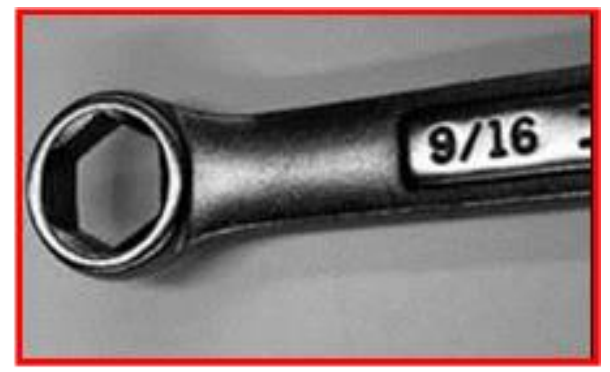

Figura 2. Medida de una llave de corona en pulgadas. Fuente: Basic Automotive Service and Systems.

\section{Instrumento de medición}

Según el Vocabulario Internacional de Metrología de la Norma Mexicana (IMNC) NMXZ-055-IMNC-2009 los instrumentos de medición "son dispositivos destinados para realizar mediciones, solo o asociado a uno o varios dispositivos suplementarios" (pág. 26). Estos instrumentos deben de ser seleccionados de acuerdo al tipo de medición a realizar, en el presente trabajo se hará referencia al micrómetro de exteriores el cual es un instrumento de medición directa lineal.

\section{Micrómetro, Palmer o Tornillo micrométrico}

El micrómetro, Palmer o Tornillo micrométrico es un instrumento de medida directa utilizado principalmente para medir con mucha precisión, se basa en el funcionamiento de un tornillo que gira a través de una tuerca fija, el desplazamiento longitudinal es proporcional al giro de dicho tornillo, es decir, la distancia recorrida al girar una vuelta el tornillo es igual al paso de la rosca (Chávez, Mejía, \& Pacheco, 2009; Fenoll, Borja, \& Seco de Herrera, 2009). 


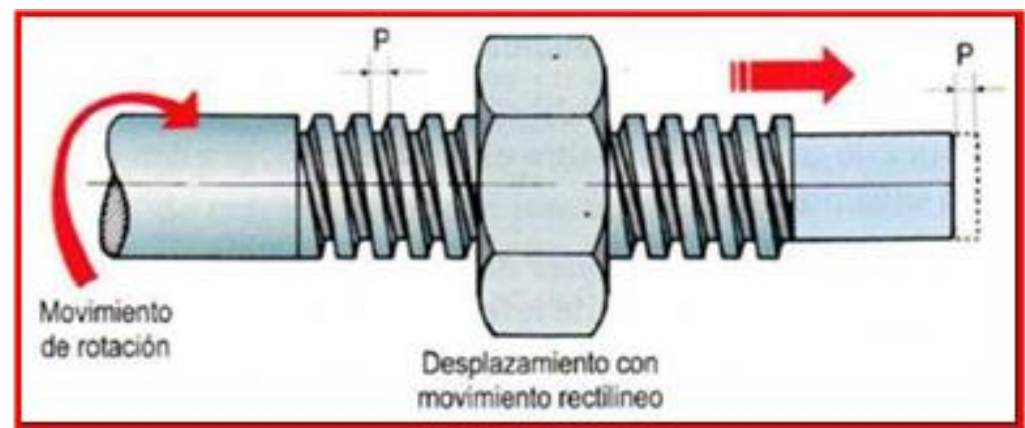

Figura 3. Principio de funcionamiento del micrómetro, tuerca-tornillo. Fuente: Mecanizado básico para electromecánica.

Las partes de un micrómetro se pueden apreciar en la siguiente figura, siendo estas: (1) trinquete; (2) tambor; (3) escala Vernier; (4) escala lineal fija; (5) cilindro; (6) contacto móvil, husillo; (7) contacto fijo, tope o yunque; (8) cuerpo del micrómetro (Fenoll, Borja, \& Seco de Herrera, 2009).

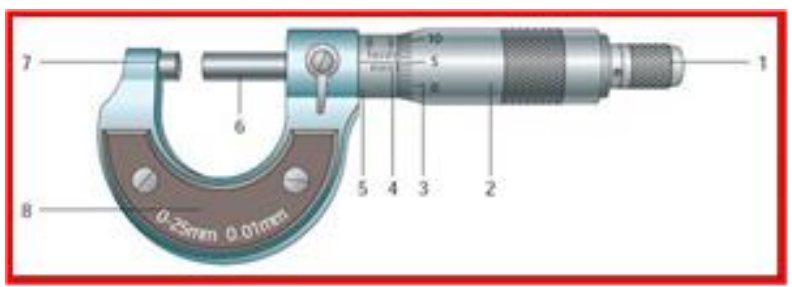

Figura 4. Partes de un micrómetro. Fuente: Mecanizado básico.

Las graduaciones de estos instrumentos de medición pueden estar en el Sistema métrico o en el Sistema Inglés de unidades. Para el Sistema métrico la escala lineal fija (figura 5) esta graduada cada $0,50 \mathrm{~mm}$ y la escala Vernier (figura 6) grabada en el tambor tiene cincuenta graduaciones, es decir, cada una equivale a $0,01 \mathrm{~mm}$. Para que el contacto móvil o husillo recorra un milímetro es necesario dar dos vueltas al tambor.

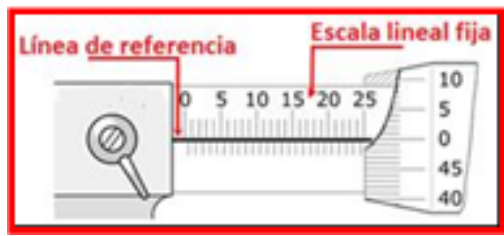

Figura 5. Escala lineal fija de un micrómetro en el Sistema métrico. Fuente: Stefanelli Eduardo.

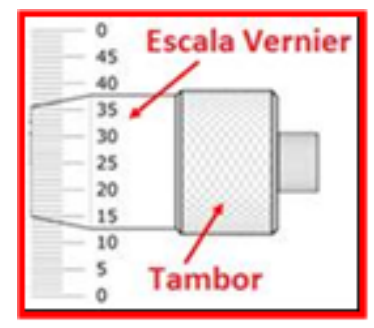

Figura 6. Escala Vernier de un micrómetro en el Sistema métrico. Fuente: Stefanelli Eduardo. 
Un micrómetro con graduaciones en el Sistema Inglés la escala lineal fija (figura 7) esta graduada cada 0.025 pulgadas, es decir, una pulgada está dividida en cuarenta partes. La escala Vernier (figura 8) grabada en el tambor tiene veinticinco partes, cada parte equivale a 0.001 pulgadas.

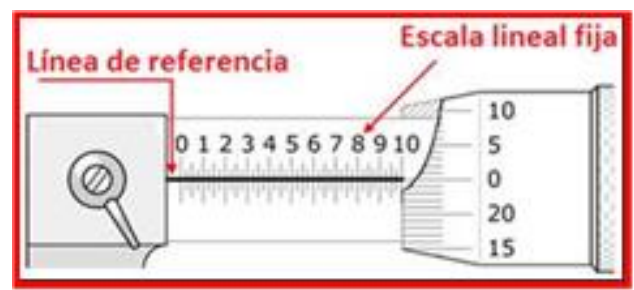

Figura 7. Escala lineal fija de un micrómetro en el Sistema Inglés.

Fuente: Stefanelli Eduardo.

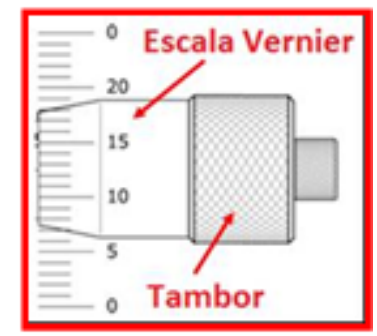

Figura 8. Escala Vernier de un micrómetro en el Sistema Inglés.

Fuente: Stefanelli Eduardo.

\section{Resolución o apreciación del instrumento de medición}

La resolución o apreciación del instrumento de medición se la puede obtener con la siguiente ecuación (Chávez, Mejía, \& Pacheco, 2009; Fenoll, Borja, \& Seco de Herrera, 2009; García \& Fernández-Bravo, 2010):

$$
\text { Resolución o Apreciación }=\frac{\text { Menor división de la escala lineal fija }}{\text { Número de división de la escala Vernier }}=\frac{d}{n}
$$

Aplicando la ecuación, se toma la menor división de la escala lineal fija de la figura 7 , $\mathrm{d}=0,025$ pulgada y el número de divisiones de la escala Vernier de la figura $8, \mathrm{n}=25$, resultando:

$$
\text { Resolución o Apreciación }=\frac{d}{n}=\frac{0,025 \text { pulgada }}{25}=0,001 \text { pulgada }
$$

\section{Aprendizaje significativo}

Autores como Ausubel (1963, 1968, 2000) y Ausubel, Novak y Hanesian (1987) han hecho una distinción muy clara entre el aprendizaje como repetición mecánica y el aprendizaje significativo, en el aprendizaje por repetición el estudiante recibe nuevos conocimientos que luego no incorporan a su estructura cognoscitiva; mientras que el aprendizaje significativo integra el nuevo conocimiento al conocimiento previo que tiene el estudiante, algunas características del aprendizaje significativo según González (2010) son: 
- Incorporación no arbitraria, sustantiva y no literal de los nuevos conocimientos

- Esfuerzo intencionado por relacionar los nuevos conocimientos con conceptos de mayor orden

- Aprendizaje relacionado con experiencias, con hechos u objetos

- Compromiso para relacionar los nuevos conocimientos con lo aprendido anteriormente

Para Ausubel, según González (2010), un aprendizaje significativo eficiente y eficaz requiere de:

- Una estructura cognitiva apropiada en el alumno, conocimiento previo.

- Materiales de aprendizaje significativos, conceptualmente transparentes, planificación adecuada.

- Una disposición favorable por parte del alumno hacia este tipo de aprendizaje, motivación.

\section{Metodologías activas para el aprendizaje}

Las metodologías activas de aprendizaje contribuyen a alcanzar un aprendizaje significativo del estudiante (De las Morenas, De la Cruz, León, Martínez, Zarate \& Carrasco, 2015), entre sus ventajas según Escribano, 2010; Rodríguez, 2011; Rodríguez, 2004 y Miguel, 2006, citado por (Rodríguez \& Larruscain, 2015) están: “mayor motivación, retención de conocimientos adquiridos, interés e implicación de los estudiantes, desarrollo de habilidades y competencias profesionales y una mayor conexión entre la teoría y la aplicación, entre el conocimiento previo y el que se va aprendiendo" (pág. 802). Para Gargallo (2009) citado por Rodríguez, Maya, \& Jaén (2012) con el uso de las metodologías activas "se desea fomentar y garantizar un compromiso individual, logrando que los alumnos sean protagonistas de su aprendizaje y lo construyan formándose criterios propios" (pág. 129).

Entre las metodologías activas para el aprendizaje están las siguientes:

- Trabajo cooperativo

- $\quad$ Aprendizaje basado en problemas

- $\quad$ Aprendizaje basado en proyectos

Con las metodologías descritas anteriormente se pretende trabajar en el desarrollo de las siguientes competencias transversales (De las Morenas, et al, 2015): Planificación y gestión del tiempo, Utilización de las TIC's, Gestión de la información (búsqueda, selección e integración), Resolución de problemas, Razonamiento crítico, Habilidades en las relaciones interpersonales, Capacidad de aplicar los conocimientos a la práctica, Creatividad, Responsabilidad, Autoconfianza.

\section{Trabajo cooperativo}

Esta metodología de aprendizaje se articula en torno al trabajo en equipo, según el Servicio de Innovación Educativa, SIE (2008) citado por Ballester (2015) "los alumnos trabajan conjuntamente para lograr determinados objetivos comunes de los que son responsables todos los miembros del equipo" (pág. 837), con el objetivo de que los estudiantes alcancen el aprendizaje 
relacionado con el contenido de la asignatura, guiados por el docente quien conforma los grupos, explica las actividades a realizar y realiza seguimiento durante el desarrollo de las mismas.

La estructura cooperativa puede orientar el trabajo en el aula y se caracteriza según Benítez \& Martínez (2014) "porque una persona alcanza su meta si el resto de personas con las que trabaja alcanzan la suya; cada uno se hace responsable de su aprendizaje y del de los demás" (pág. 34), de esta forma se logra que los estudiantes trabajen juntos y aprovechen al máximo el aprendizaje propio y entre sí. Como estrategia, el aprendizaje cooperativo inicia un cambio real en la forma de relación entre estudiantes y docente-estudiante, construyendo de manera colectiva el conocimiento y el desarrollo de habilidades mixtas (aprendizaje, desarrollo personal y social).

Según Benítez \& Martínez (2014) el aprendizaje cooperativo:

También se sostiene en la aportación de Vigotsky (1984) respecto a que el psiquismo humano se forma en la actividad y la comunicación a partir de algunas premisas básicas innatas. Actividad y comunicación son dos aspectos que determinan el nivel de desarrollo del psiquismo, entre ellos la inteligencia y la creatividad" (pág. 26).

\section{Aprendizaje basado en problemas}

Esta metodología conocida como Problem-Based Learning (PBL), busca que los alumnos construyan su conocimiento sobre problemas o casos de la vida real planteados por el docente y guiados en los siguientes pasos: primero se presenta el problema, se identifica las necesidades de aprendizaje, se busca la información necesaria y nuevamente se regresa al problema, la finalidad de esto es obtener una formación en competencias profesionales con el objetivo de estimular el liderazgo, la comunicación, la toma de decisiones, la crítica, la creatividad y el trabajo multidisciplinar. El estudiante debe de analizar el problema y empezar a realizar una lluvia de ideas que le permita obtener un listado de lo que conoce y desconoce y otra con lo que necesita para resolverlo, lo más importante definir el problema para poder realizar una búsqueda adecuada de información (Trejo, Ramírez, Mata, \& Hanafi, 2014).

Esta metodología se puede desarrollar de manera individual o en grupo, tratando de crear un ambiente similar al real, trabajando e interactuando entre ellos, intercambiando conocimientos mientras, el papel del docente queda como motor de aquello que el estudiante debe de hacer y sin dejar a un lado recursos como las clases magistrales, seminarios, conferencias, obtención de información, aportaciones de profesionales del área y la ayuda del tutor.

Aprendizaje basado en proyectos

Según Yadarola (2012, pág. 141) "se aprende a ser ingeniero actuando como ingeniero", por lo cual, al estudiante universitario desde sus inicios se le debe enfrentar a un aprendizaje activo por medio de proyectos de ingeniería simples (lo más real posible), que les permita experimentar situaciones propias de un ingeniero dejando a un lado el exceso de teoría y dando paso a la práctica. Este enfoque pedagógico aconsejado por la UNESCO, nacido en la Universidad de Dinamarca requiere de docentes que "actuando como guías o facilitadores, puedan aumentar la motivación y el interés de los estudiantes para resolver problemas reales y aprender por descubrimiento" (Yadarola, 2012, pág. 
141). Su finalidad es la de cumplir con los objetivos de aprendizaje relacionados con los contenidos del programa de la asignatura elegida. El docente puede participar y dirigir el trabajo de los estudiantes.

Un proyecto puede acelerar el aprendizaje, aumentando la creatividad con el fin de buscar una posible solución, atendiendo a ciertas restricciones que se podrían presentar, tales como son las económicas, técnicas, sociales, ambientales y muchas veces situaciones desconocidas, lo cual exige: "considerar alternativas, tomar decisiones, desarrollar la capacidad de análisis y síntesis y el espíritu crítico, mayor responsabilidad por el resultado, aceptar el hábito de trabajar en equipo muchas veces interdisciplinario, ser emprendedores" (Yadarola, 2012, págs. 141-142).

Tabla 1. Objetivos específicos de la asignatura y su relación con los productos finales del proyecto.

\begin{tabular}{ll}
\hline Objetivos específicos de la asignatura & Productos finales del proyecto \\
\hline $\begin{array}{l}\text { Identificar las unidades básicas y derivadas } \\
\text { del Sistema Internacional de unidades y del } \\
\text { sistema inglés, así como su simbología y } \\
\text { reglas de uso general }\end{array}$ & $\begin{array}{l}\text { Conocer los sistemas de medidas y unidades } \\
\text { empleados en la actualidad. }\end{array}$ \\
$\begin{array}{l}\text { Identificar las características de los diferentes } \\
\text { instrumentos de medición. }\end{array}$ & $\begin{array}{l}\text { Conocer el instrumento de medición, sus } \\
\text { partes, tipos de medidas que se pueden } \\
\text { realizar y las apreciaciones o resoluciones del } \\
\text { mismo. }\end{array}$ \\
$\begin{array}{l}\text { Realizar mediciones lineales mediante el } \\
\text { empleo del instrumento, teniendo en cuenta }\end{array}$ & $\begin{array}{l}\text { Utilizar interpretar y mantener de manera } \\
\text { correcta el instrumento de medición. }\end{array}$ \\
$\begin{array}{l}\text { las normas y procedimientos establecidos } \\
\text { con este propósito. }\end{array}$ & \\
\hline
\end{tabular}

Descripción del método para lograr un aprendizaje significativo en la lectura del micrómetro en el Sistema métrico de unidades

El objetivo del presente trabajo es lograr fomentar la actitud creativa de los estudiantes de la asignatura Metrología, específicamente con el aprendizaje del instrumento de medición directa denominado micrómetro de exteriores, mediante actividades de aprendizaje basados en el análisis y resolución de problemas, y trabajo en equipo. Los cuales, buscan implicar mucho más a los estudiantes en su aprendizaje, mediante su constante participación de manera activa y creativa. Además, se ha optado por reconfigurar las clases magistrales haciendolas mucho más participativas, haciendo que los estudiantes tomen el control de su aprendizaje.

Uno de los inconvenientes es que los estudiantes no logran conectar el aprendizaje previo (clases magistrales) con las prácticas de lectura e interpretación del micrómetro, esto se da por la no memorización (aprendizaje mecanicista) del valor de las graduaciones representadas en la escala lineal fija y en la escala Vernier, sean estas del Sistema métrico o del Sistema Inglés de unidades, según Martín (2010) el: 
Aprendizaje significativo y el aprendizaje mecanicista no son siempre dos tipos opuestos de aprendizaje, sino que pueden complementarse durante el proceso de enseñanza y darse simultáneamente en la misma tarea de aprendizaje. Por ejemplo, la memorización de las tablas de multiplicar es necesaria y formaría parte del aprendizaje mecanicista, mientras que su uso en la resolución de problemas correspondería al aprendizaje significativo (pág. 79).

Pero no solo se aprende registrando datos en el cerebro, también se aprende construyendo (participación de manera activa), haciendo que lo aprendido sea más duradero e integrador, además de que sea autónomo y estimulante para el estudiante, que pueda comprender lo que el docente expone en clases, de esta manera la información se incorpore a su estructura mental y pase a formar parte de la memoria comprensiva.

Por tal razón, se ha optado por aplicar el principio de funcionamiento del micrómetro mediante la elaboración de la escala lineal fija y la escala Vernier ampliada, utilizando un elemento roscado (exterior e interior) M8x1, 25 cuya longitud depende de la longitud del material (madera en este caso) utilizado para fabricar el cilindro y el tambor sobre el cual se realizaran las graduaciones.

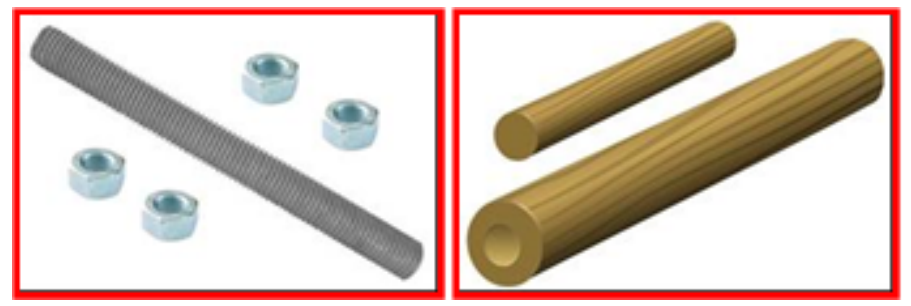

Figura 9. Elementos roscados M8x1, 25 y cilindros de madera.

Los elementos roscados se acoplan a cada cilindro de madera como se muestra en la figura 10, en la parte móvil (tambor) se ensambla el elemento roscado exterior (tornillo) y el elemento roscado interior (tuerca) en lo que sería el cilindro sobre el cual se encuentra la escala lineal fija.

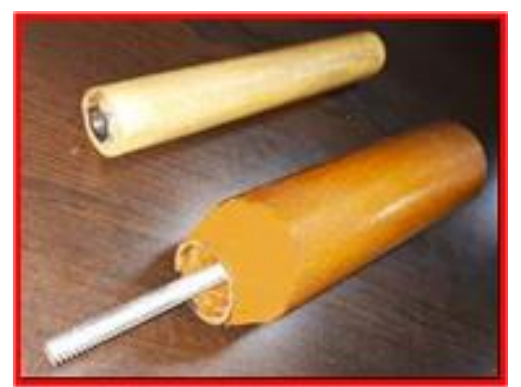

Figura 10. Ensamble de los elementos roscados en los cilindros de madera.

Luego se procede a realizar una línea en el cilindro donde se acopló la tuerca a la cual llamaremos línea de referencia y una línea en el tambor con el número cero (figura 11), con esto se procederá a realizar una graduación por cada vuelta que del tambor sobre la línea se referencia, tal como se muestra en la figura 12. 


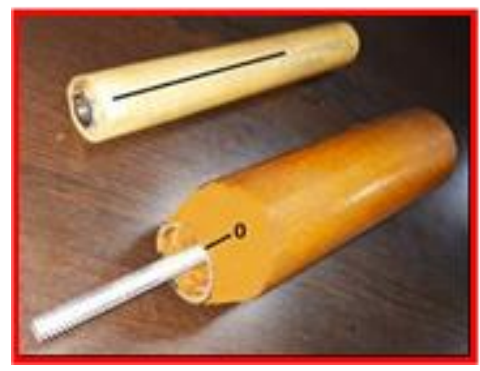

Figura 11. Trazado de la línea de referencia y la línea cero.

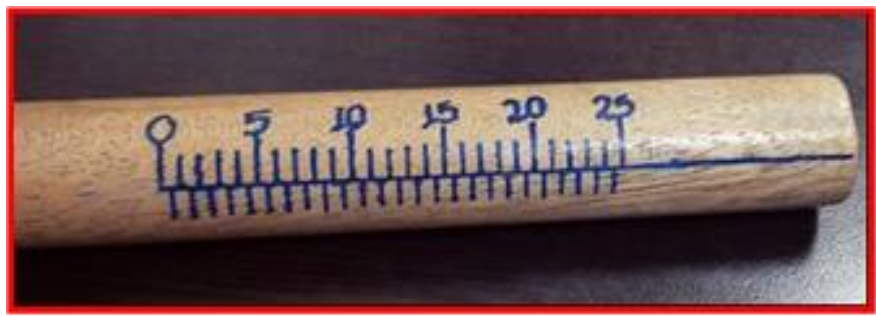

Figura 12. Escala lineal fija grabada en el cilindro.

Ahora se necesita realizar las graduaciones de la escala Vernier, para esto se tiene que conocer la longitud de la circunferencia donde se realizará la escala, con la ayuda de la siguiente ecuación (Ortiz Campos, Ortiz Cerecedo, \& Ortiz Cerecedo, 2017):

Donde:

$\mathrm{C}=$ longitud de la circunferencia

$\mathrm{d}=$ diámetro

En este caso se tiene un diámetro de $26 \mathrm{~mm}$, reemplazando en la ecuación dos, se obtiene:

La longitud de circunferencia se la tiene que dividir en cincuenta partes iguales, es decir, $82 \mathrm{~mm} / 50$ partes $=1,64 \mathrm{~mm} /$ parte. Para obtener esto primero se traza el segmento AB y a un extremo se traza una línea $\mathrm{BC}$ perpendicular al segmento $\mathrm{AB}$ de unos $65 \mathrm{~mm}$ de longitud, luego se traza una línea AC' de $100 \mathrm{~mm}$. Al segmento AC' se la divide en cincuenta partes iguales, es decir, se realiza un trazo cada dos milímetros tal como se muestra en la figura 13. También se traza dos paralelas al segmento $\mathrm{AB}$ con una separación de 5 y $8 \mathrm{~mm}$.

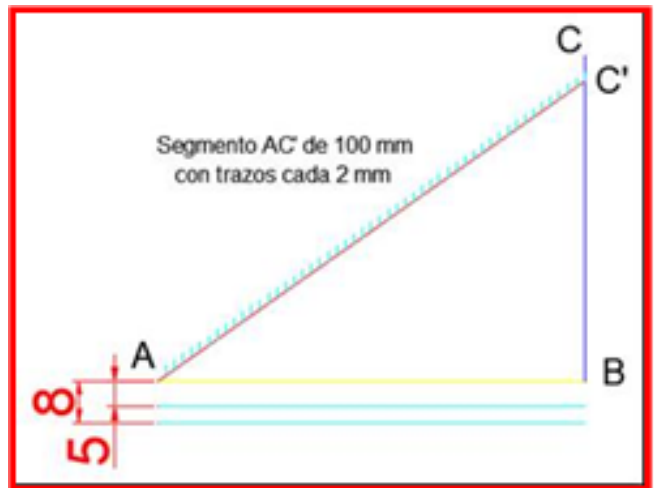

Figura 13. Triángulo ABC' para realizar la escala Vernier con 50 partes iguales. 
Con la ayuda de una escuadra y una regla se procede a trazar líneas desde el segmento AC' hasta las líneas paralelas al segmento $\mathrm{AB}$, simulando las graduaciones de la escala Vernier como se mostró en la figura 6.

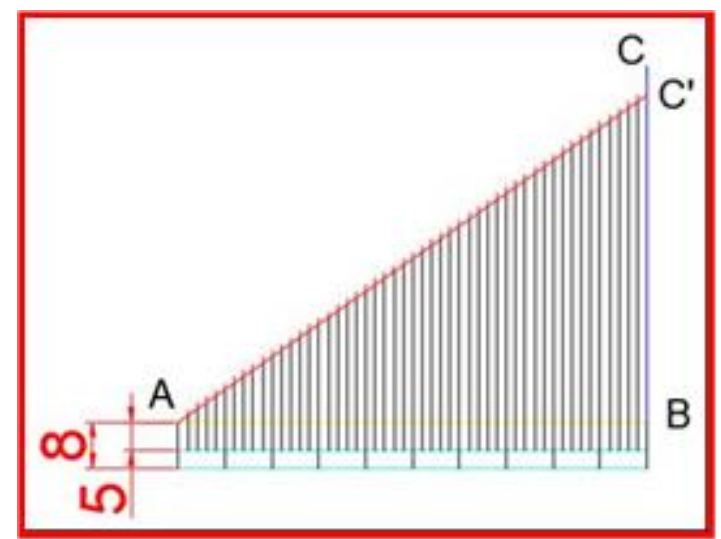

Figura 14. Representación de los trazos cada dos milímetros.

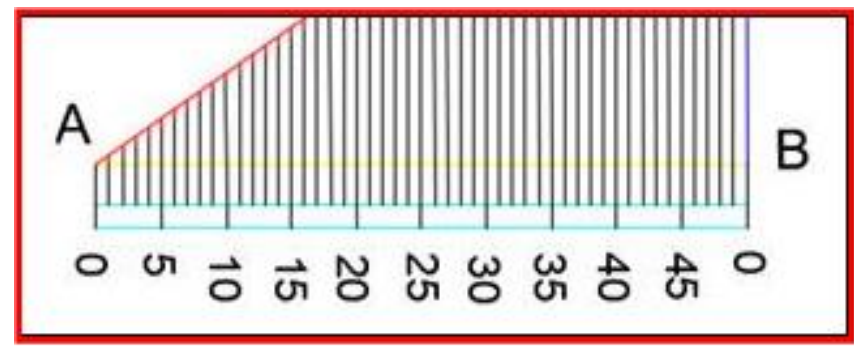

Figura 15. Numeración de las líneas simulando la escala Vernier.

La escala Vernier representada en la figura 15 se la realiza o graba en el extremo del cilindro de madera que será el tambor del micrómetro.

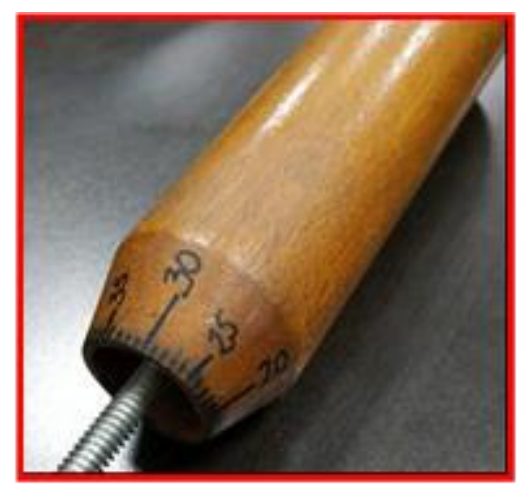

Figura 16. Representación de las graduaciones en el tambor de la escala Vernier. 


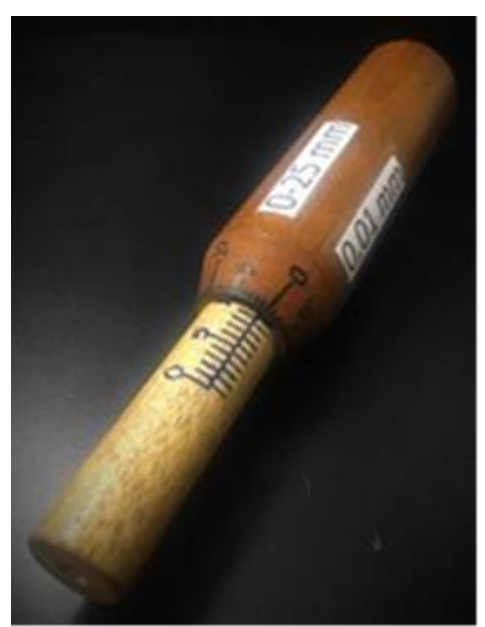

Figura 17. Cilindro y tambor ampliado de un micrómetro en centésimas de milímetros $(0,01)$.

\section{Discusión}

El aprendizaje significativo se da de manera experiencial a través de hechos y conocimientos previos del aprendiz y de manera social, es decir, por la interacción con otras personas, según Moreno (2014), las teorías de los esquemas cognitivos de Piaget y el aprendizaje significativo de Ausubel, han hecho énfasis en la experiencia previa del ser humano como punto de partida para desarrollar el conocimiento, mientras que para Vygotsky, según Gutiérrez y Rada (2012), el conocimiento se da producto de la interacción del sujeto y el medio (social y cultural), en otras palabras el conocimiento es el resultado de la relación del sujeto con el contexto desde lo social y lo cultural.

Basados en esto se ha optado por conformar grupos de trabajo cooperativos para el desarrollo de la aplicación del principio de funcionamiento y ampliación de la escala lineal fija y la escala Vernier de un micrómetro en el Sistema métrico de unidades $(0,01 \mathrm{~mm})$, con la finalidad de involucrar al estudiante en su formación, guiados por el docente quien a su vez cumple con su rol de facilitador. En este trabajo, a la vez que el estudiante aplica los conocimientos impartidos por el docente, brinda al estudiante un medio con el que pueda realizar prácticas de medición que luego las consolida con la práctica utilizando el instrumento, micrómetro, real. 


\section{Conclusiones}

Las medidas con las cuales se quiera trabajar en las ampliaciones de cada una de las escalas queda en consideración del lector de este trabajo, debiendo empezar por la selección del elemento roscado a utilizar (diámetro y paso) ya que esto servirá para determinar la dimensiones del cilindro y del tambor a los que luego se les grabará las graduaciones.

Con este trabajo se ha demostrado que haciendo que el estudiante se involucre en su proceso de formación se logra que el aprendizaje sea significativo y duradero por medio de un aprendizaje activo y cooperativo.

Como es un trabajo de ampliación de las graduaciones de las distintas presentaciones de un micrómetro se puede optar por numerar cada una de las líneas o divisiones de la escala lineal fija y de la escala Vernier, para facilitarles el aprendizaje de la interpretación y lectura de medidas, así como la elaboración de micrómetro de madera en el cual el estudiante realice las graduaciones tanto para el sistema métrico como para el sistema inglés de unidades. 


\section{Bibliografía}

Ballester, E. (2015). Metodologías docentes y de evaluación para la formación en competencias en la asignatura de "Eficiencia energética de instalaciones térmicas. En M. Mata, Innovación educativa en las enseñanzas técnicas (Vol. II, págs. 833-845). España: Ediciones de la universidad de Castilla-La Mancha.

Benítez, L., \& Martínez, R. (2014). La cooperación y colaboración en la educación superior tecnológica: un paradigma en construcción. En R. Martínez, M. Villanueva, \& E. Canales, Aprendizaje cooperativo: una alternativa para mejorar la instrumentación didáctica en la Educación Superior Tecnológica (págs. 21-38). México: Ediciones Díaz de Santos.

Chávez, F., Mejía, R., \& Pacheco, G. (2009). Introducción a la metrología dimensional. México: Instituto Politécnico Nacional.

De las Morenas, J., De la Cruz, J., León, S., Martínez, F., Zarate, R., \& Carrasco, M. (2015). Experiencia de colaboración docente entre I.E.S. Mercurio y la E.I.M.I.A en la construcción del prototipo de un vehículo eléctrico. En M. Mata, Innovación educativa en las enseñanzas técnicas (Vol. II, págs. 777-787). España: Ediciones de la Universidad de Castilla-La Mancha.

Del Campo, M., \& Robles, J. (2012). La metrología, motor de innovación tecnológica y desarrollo industrial. e-medida, 1(1), 29-41. Obtenido de http://www.emedida.com/documentos/Numero-1/articulos

Escamilla, A. (2014). Metrología y sus aplicaciones. México: Grupo Editorial Patria.

Fenoll, J., Borja, J., \& Seco de Herrera, J. (2009). Mecanizado básico. Madrid: Mac Millán Iberia S.A.

García, J., \& Fernández-Bravo, P. (2010). Mecanizado básico. Madrid: Paraninfo.

González, F. (2010). El Mapa Conceptual y el Diagrama UVE. Recursos para la Enseñanza Superior en el siglo XXI. Madrid: Narcea S.A. de Ediciones.

Herrero, I., García, C., González, E., Molina-Tanco, L., Pérez, E., \& Urdiales, C. (2013). Aprendizaje cooperativo en el ámbito de la Ingeniería: una experiencia de iniciación al Trabajo en Grupo. Revista de docencia Universitaria, 11(Número especial dedicado a Engineering Education), 221-251. Obtenido de http://red-u.net

Instituto Mexicano de Normalización y Certificación, A.C. (2013). Vocabulario Internacional de Metrología-Conceptos fundamentales y generales, y términos asociados (VIM). NMX-Z055-IMNC-2009. Obtenido de Catálogo de Normas: https://www.imnc.org.mx/

Martín, F. (2010). Fomentar el aprendizaje. En D. Cervera, Didáctica de la tecnología (págs. 7792). España: GRAÓ. 
Ortiz Campos, F. J., Ortiz Cerecedo, F. J., \& Ortiz Cerecedo, F. J. (2017). Matemáticas 2. México: Grupo Editorial Patria, S.A.

Owen, C. (2011). Basic Automotive Service and Systems. Canadá: Delmar, Cengage Learning. Raghavendra, N., \& Krishnamurthy, L. (2013). Enginnering metrology and measurements. India: Oxford University Press.

Rodríguez, A., \& Larruscain, J. (2015). Metodologías activas PBL en la asignatura Sistema de Gestión Integrada. En M. Mata, Innovación educativa en las enseñanzas técnicas (Vol. II, págs. 801-808). España: Ediciones de la Universidad de Castilla-La Mancha.

Rodríguez, K., Maya, M., \& Jaén, J. (2012). Educación en Ingenierías: de las clases magistrales a la pedagogía del aprendizaje activo. En Ingeniería y desarrollo (págs. 125-142). Barranquilla: Universidad del Norte.

Stefanelli, E. (2017). Metrología. Obtenido de Micrómetro en centésimas de milímetro - uso, lectura e interpretación: https://www.stefanelli.eng.br/es/micrometro-centesimasmilimetro-uso/

Trejo, J., Ramírez, E., Mata, F., \& Hanafi, I. (2014). La experiencia del aprendizaje basado en problemas y trabajos proyectuales en las asignaturas de ingeniería de estructuras en la Escuela de Ingeniería de Almadén. En M. Mata, Innovación educativa en las enseñanzas técnicas (Vol. II, págs. 615-627). España: Ediciones de la Universidad de Catilla-La Mancha.

Yadarola, M. (2012). Cambios de modelos en la formación y práctica de la ingeniería. Buenos Aires: ANI - Academia Nacional de Ingeniería. 